\title{
Consenso sobre manejo integral del neonato con encefalopatía hipóxico isquémica
}

\author{
JOSÉ M. NOVOA P. ${ }^{1,2,3}$, MARCELA MILAD A. ${ }^{1,4,5}$, JORGE FABRES B..$^{6,7,8}$, JUAN A. FASCE C..$^{9,10}$, \\ PAULINA A. TOSO M. ${ }^{7,8}$, MANUEL ARRIAZA O. ${ }^{11,12}$, M $^{\mathrm{a}}$ CAROLINA GANDOLFI E. ${ }^{13}$, \\ M MARGARITA SAMAMÉ M. ${ }^{1,14,15}$, CARLOS ASPILLAGA M. ${ }^{1,16}$. \\ 1. Directorio Rama de Neonatología, Sociedad Chilena de Pediatría. \\ 2. Jefe Neonatología Hospital Padre Hurtado. \\ 3. Profesor Departamento. Pediatría Facultad Medicina Clínica Alemana/Universidad del Desarrollo. \\ 4. Jefe Neonatología Clínica Santa María. \\ 5. Profesor Auxiliar Facultad de Medicina Universidad de los Andes. \\ 6. Presidente Rama de Neonatología, Sociedad Chilena de Pediatría. \\ 7. Profesor Asistente de Pediatría, Pontificia Universidad Católica de Chile. \\ 8. Neonatólogo Hospital Clínico Pontificia Universidad Católica de Chile. \\ 9. Neonatólogo Hospital Regional de Concepción. \\ 10. Profesor Asistente Departamento Pediatría, Facultad Medicina Universidad de Concepción. \\ 11. Neonatólogo y Neurólogo Infantil Hospital Dr. Sótero del Río. \\ 12. Instructor Adjunto de Pediatría, Pontificia Universidad Católica de Chile. \\ 13. Neonatóloga Clínica Santa María. \\ 14. Neonatóloga Hospital San Juan de Dios. \\ 15. Profesora Agregada, Departamento Pediatría y Cirugía Infantil, Campus Occidente, Universidad de Chile. \\ 16. Profesor Asistente de Pediatría Facultad de Medicina Universidad de Chile, Departamento de Obstetricia, \\ Ginecología y Neonatología Hospital Clínico San Borja Arriarán.
}

\section{ABSTRACT \\ Consensus statement on integral management of the newborn with hypoxic-ischemic encephalopathy}

Neonatal encephalopathy secondary to birth asphyxia, the hypoxic ischemic encephalopathy, remains a major cause of postnatal death and neurological sequelae worldwide. Supportive therapy has been the mainstay of treatment. Recently series of multicenter clinical trials show the benefits of therapeutic hypothermia in this high risk population. The International Liaison Committee on Resuscitation (ILCOR) has recommended hypothermia as a standard of care and a beneficial therapy using the protocols followed in large clinical trials. Our objective was to develop a practical guide to be used at a national level in Chile, compatible with published protocols and standardized on an international basis, practical and compatible with the country's situation, and considering an integral management of the asphyxiated neonates, rescue and neuroprotective therapies. In summary, to establish rescue therapies, with the aim objective of support measures that promote cerebral and systemic oxygenation/perfusion, ensuring optimal cellular homeostasis, which are the basis of the rescue measures in asphyxiated neonates. Along with these rescue therapies, the objective is to perform the more beneficial neuroprotective therapy known today, hypothermia. Hypothermia should be conducted in a level 3 Neonatal Intensive Care Unit, with multidisciplinary care and standardized protocols and also multidisciplinary follow-up and rehabilitation therapies.

(Key words: Perinatal asphyxia; Hypoxic ischemic encephalopathy, Therapeutic hypothermia, neonatal asphyxia, Neuroprotection, Newborn, neonate).

Rev Chil Pediatr 2012; 83 (5): 492-501

Recibido el 17 de agosto de 2012, aceptado para publicación el 3 de septiembre de 2012.

Correspondencia a:

Dr. José Manuel Novoa P.

E-mail: jnovoap@yahoo.es 


\section{RESUMEN}

La encefalopatía neonatal, secundaria a asfixia durante el parto, encefalopatía hipóxico isquémica, sigue siendo una importante causa de muerte posnatal y déficit neurológico permanente en todo el mundo. La terapia de apoyo ha sido el pilar del tratamiento. Recientemente grandes ensayos clínicos han demostrado los beneficios de la hipotermia terapéutica en esta población de alto riesgo. La International Liaison Committee on Resuscitation (ILCOR: Comité Internacional de Coordinación en Resucitación) recomienda la hipotermia como un estándar de cuidado como terapia beneficiosa. Nuestro objetivo fue desarrollar una guía práctica de recomendación de manejo a nivel nacional, compatible con los protocolos internacionales, práctico y adecuado a la situación del país, que considere la terapia de rescate y de neuroprotección. Instaurar las terapias de rescate, generando todas las medidas de apoyo que favorezcan la oxigenación y perfusión cerebral y sistémica, asegurando una óptima homeostasis celular, y sobre éstas instaurar la medida de neurorescate más beneficiosa conocida hasta hoy, que es la hipotermia moderada. Ésta debe llevarse a cabo en Unidades de Neonatología de nivel terciario con capacidad de atención multidisciplinaria y con protocolos estandarizados. Estos centros deben ofrecer un seguimiento multidisciplinario protocolizado y terapias de rehabilitación.

(Palabras clave: Asfixia perinatal; Encefalopatía hipóxico-isquémica; Hipotermia terapéutica; Neuroprotección; Recién nacido, Neonato).

Rev Chil Pediatr 2012; 83 (5): 492-501

\section{Introducción}

La encefalopatía hipóxico-isquémica (EHI), sigue siendo uno de los más importantes problemas médicos perinatales de todo el mundo. La incidencia de EHI en los países desarrollados es de aproximadamente 1-2 casos por cada 1000 nacidos vivos ${ }^{1}$. En Chile se dispone de pocos registros ${ }^{2}$, algunos de ellos resúmenes de congresos científicos, pudiendo estimar que la incidencia de EHI en todos sus grados es de 4 a 6 casos por 1000 nacidos vivos y los casos moderados y severos de 2 a 3 por mil.

La EHI es una importante causa de muerte neonatal y de discapacidad permanente del desarrollo neurológico, evidenciado por diferentes grados de parálisis cerebral y/o secuelas neurosensoriales. En las últimas décadas se han realizado muchos esfuerzos para mejorar el manejo y pronóstico de la EHI. Recientemente, una serie de estudios clínicos ${ }^{3-6}$ han demostrado que la hipotermia terapéutica iniciada antes de las $6 \mathrm{~h}$ de vida mejora el resultado a los 18 meses de seguimiento de los neonatos que cursan con encefalopatía moderada a severa.

Un meta-análisis reciente $^{7}$, que incluye los estudios CoolCap ${ }^{3}, \mathrm{NICHD}^{4}$, $\mathrm{TOBY}^{5}$, demostró que la hipotermia moderada se asocia con una reducción en un $11 \%$ en la muerte y discapacidad severa a los 18 meses (RR 0,81, IC 95\% 0,71-0,93, $p=0,002$ ). La hipotermia aumentó la sobrevida con función neurológica normal en un $12 \%$ (RR 1,53, IC 95\% 1,22$1,93, \mathrm{p}=0,001)$ y redujo la discapacidad grave en los sobrevivientes $(\mathrm{p}=0,006)$, parálisis cerebral $(p=0,004), y$ MDI/PDI $<70(p=0,01$ y $\mathrm{p}=0,02$, respectivamente). Esta evidencia acumulada ha llevado a la incorporación de la hipotermia en la práctica clínica como terapia clínica reconocida.

En 2010, el Comité Internacional de Coordinación en Resucitación (ILCOR) y la American Heart Association (AHA) han recomendando la hipotermia para el manejo de los neonatos con EHI moderada a grave ${ }^{8}$. La Rama de Neonatología de la Sociedad Chilena de Pediatría, se hace partícipe de esta recomendación.

La hipotermia ha sido introducida en Chile en muchas unidades como parte del tratamiento de la EHI, no existiendo un protocolo consensuado de hipotermia. Por lo tanto, es necesario desarrollar un Protocolo de Manejo del RN con EHI que sea de consenso nacional sobre la base de la evidencia científica disponible para el manejo integral de estos pacientes y considerando que el manejo debe realizarse en centros terciarios.

Debemos considerar que el diagnóstico de EHI sólo es aplicable a RN de término o cerca- 
no a término, dado que el fenómeno hipóxico isquémico afecta la circulación crítica que en esta población característicamente corresponde a la región cortical y subcortical cerebral, expresándose con síntomas de encefalopatía. En prematuros la lesión por la misma causa es principalmente periventricular, expresándose la asfixia del prematuro como leucomalacia periventricular?.

Esta recomendación sobre Manejo Integral del neonato con encefalopatía hipóxico isquémica, se realiza incluyendo terapia de rescate, terapia de neuroprotección, traslado, neuroimágenes y seguimiento.

\section{Terapia de rescate}

Establecer todas las medidas de apoyo que favorezcan la oxigenación y perfusión cerebral y sistémica, asegurando óptima homeostasis celular, es la base de las medidas de rescate del neonato asfixiado ${ }^{10,11}$.

Se debe considerar en la etapa de reanimación y estabilización inicial del neonato asfixiado, la reposición de volumen inicialmente con solución fisiológica. La hipovolemia se presenta debido a una condición de vasocontricción fetal como fenómeno compensatorio a la hipoxia intrauterina, a lo que se suma la frecuente ligadura precoz de cordón determinada por la condición de nacimiento, por lo que es fundamental corregir precozmente la volemia.

Conceptualmente, siempre hay que actuar considerando que un RN asfixiado puede presentar alteración de autorregulación cerebral ${ }^{9}$, por lo tanto, el paciente se debe enfrentar asumiendo que la perfusión cerebral está reflejada directamente por la presión arterial de un modo pasivo. Es objetivo fundamental de la terapia establecer medidas para mantener presión arterial en rango normal para su edad.

En cuadros asfícticos de importancia, es casi de regla la disfunción miocárdica con compromiso de la contractibilidad miocárdica. Estudios han demostrado que la fracción de acortamiento de ventrículo izquierdo (FaVi) en $\mathrm{RN}$ asfixiado oscila en promedio entre 22$25 \%{ }^{12}$, lo cual determina necesidad de apoyo precoz con inotrópos que aseguren óptima contractibilidad $^{13-15}$.
El manejo óptimo de estos pacientes se logra mediante evaluación con ecocardiografía funcional que permite estimar volumen de llenado ventricular y contractibilidad, adecuando el aporte de volumen y la dosis de vasoactivos. En general, si no se dispone de ecocardiografía funcional, iniciar con dopamina $5 \mathrm{mcg} / \mathrm{kg} /$ min, subiendo hasta $12 \mathrm{mcg} / \mathrm{kg} / \mathrm{min}$, adicionar dobutamina si persiste falla miocárdica. Tener precaución que dosis mayores de dopamina aumentan la resistencia vascular periférica, con ello el trabajo miocárdico y resistencia vascular pulmonar, condicionando el aumento de presión de arteria pulmonar. Por otra parte, considerar que la dobutamina mejora la contractibilidad, no así la presión arterial ${ }^{14,15}$.

En neonatos de término, que al nacer requieren reanimación con ventilación con presión positiva, lo adecuado es comenzar con aire en lugar de oxígeno al 100\%, para evitar daño por hiperoxia. Si a pesar de una ventilación efectiva no hay un aumento en la frecuencia cardíaca o si la oxigenación, medida por oximetría de pulso medida en extremidad superior derecha, sigue siendo inadecuada, se debe considerar el uso de una mayor concentración de oxígeno $^{8}$. El nivel de oxigeno se debe mantener dentro de límites normales. Evitar hipoxia o hiperoxia. Se debe manejar según objetivo de saturación medida preductal ${ }^{8}$. Los niveles de $\mathrm{pCO}_{2}$ deben mantenerse en rangos normales (35-45 mm Hg) $)^{9}$, se debe considerar cambio en el rango de nivel de $\mathrm{pCO}_{2}$ en caso de establecerse hipotermia.

La perfusión se ve alterada por presencia de hiperviscosidad o anemia. Se debe mantener hematocrito entre $45-60 \%$. En caso de hematocrito bajo el rango se corregir con transfusión de glóbulos rojos en alícuotas de $15 \mathrm{ml} / \mathrm{kg}$.

La hipoglicemia es un marcador de mal pronóstico, incluso más que el Apgar y la acidosis, por lo cual es fundamental mantener desde un comienzo glicemia estable entre 75$100 \mathrm{mg}^{0}{ }^{16-17}$.

Los niveles de calcio deben mantenerse dentro de límites normales. La hipocalcemia, que es frecuente en el neonato asfixiado, puede comprometer la contractibilidad miocárdica y/o provocar convulsiones. Considerar que la corrección espontánea o activa de la acidosis 
determina baja del calcio iónico ${ }^{10}$. Es necesario corregir precozmente la hipocalcemia. Es adecuado disponer de medición de calcio iónico en los laboratorios de centros terciarios de salud para el manejo paciente grave.

En caso de oliguria $(<0,5 \mathrm{ml} / \mathrm{kg} / \mathrm{h})$ a las 4-6 h de vida siempre se debe descartar falla pre-renal. La oliguria no debe atribuirse a necrosis tubular aguda o a secreción inapropiada de hormona antidiurética, a menos que se haya excluído la etiología prerenal como hipovolemia, alteración de contractibilidad o vasodilatación periférica por disautonomía ${ }^{11}$. Estos pacientes deben permanecer con sonda vesical para medición estricta de diuresis.

En todo neonato que requiera reanimación avanzada o en el cual se sospeche va a evolucionar con signos de encefalopatía, se sugiere apagar las fuentes de calor y permitir que se enfríe en forma espontánea ${ }^{18}$. De esta forma los recién nacidos alcanzan temperatura rectal entre $34-35^{\circ} \mathrm{C}$. Esto se debe mantener hasta decidir si el paciente es candidato a hipotermia o puede seguir tratamiento habitual, permitiéndole la termorregulación entre $36,5^{\circ}$ y $37^{\circ} \mathrm{C}$.

\section{Terapia de neuroprotección: hipotermia moderada}

Se debe considerar criterios de inclusión ${ }^{3-6}$ para hipotermia a neonatos con lo siguiente: 1) Evento perinatal agudo hipóxico, como bradicardia fetal, prolapso de cordón, desprendimiento de placenta, entre otros; 2) Criterio A: $R N \geq 35$ semanas de gestación con acidosis dentro de los primeros 60 minutos de vida, definida como presencia en sangre de cordón umbilical, arterial, venosa o capilar de $\mathrm{pH}$ $<7,00$ o déficit de base $\geq 16 \mathrm{mmol} / \mathrm{L}$. Si no se dispone de $\mathrm{pH}$ considerar al menos uno de las siguientes condiciones: Apgar a los $10 \mathrm{mi}-$ nutos de $\leq 5$ o necesidad de reanimación con ventilación endotraqueal o por máscara $\geq 10$ minutos. Los RN con evento perinatal agudo hipóxico que cumplen con criterios A serán evaluados para saber si cumplen con los criterios de anormalidad neurológica de ingreso por personal capacitado; 3) Criterio B: signos de encefalopatía de moderada a severa, que consiste en estado alterado de conciencia (le- targia, estupor o coma) más al menos uno de las siguientes condiciones: hipotonía, reflejos anormales (incluyendo anormalidades oculomotoras o pupilar), succión débil o ausente y convulsiones clínicas.

En pacientes con evento perinatal hipóxico agudo que cumplen con los criterios A y que el criterio B es poco claro, cuando EEG de amplitud integrada (aEEG) está disponible, será evaluado con esta tecnología, leída por personal capacitado, que permita establecer la condición eléctrica cerebral y progresión de la encefalopatía.

En algunas ocasiones es difícil distinguir clínicamente entre EHI leve o moderada según clasificación de Sarnat y Sarnat. En esta situación si el aEEG está anormal después de $1 \mathrm{~h}$ de observación permite plantear EHI moderada con indicación de hipotermia ${ }^{19}$. La disponibilidad o interpretación de aEEG, no debe retrasar la decisión de iniciar la hipotermia, más allá del tiempo esperado de registro. A lo menos se debe disponer de 30-60 min de duración de registro de aEEG que muestre presencia de depresión de voltaje moderado (cuando el margen superior es $>10$ microvolt y el margen inferior $<5$ microvolt), depresión de voltaje severo (si el margen superior $<10$ microvolt) o actividad anormal de aEEG o convulsiones ${ }^{13,14}$. El EEG convencional multicanal no puede ser utilizado con el propósito de definir los márgenes superior e inferior de voltaje. En general, el aEEG se recomienda como monitorización y no como criterio de inclusión.

Se consideran criterios de exclusión ${ }^{3-6}$ : RN mayores de $6 \mathrm{~h}$ de vida al momento de inicio de la hipotermia (en caso de centros que no disponen de hipotermia y los pacientes deban trasladarse, este tiempo podría prolongarse hasta 8-12 h), RN con restricción severa del crecimiento intrauterino con peso al nacer inferior a 1800 gramos (este criterio utilizado en los protocolos de investigación, del punto de vista clínico no es absoluto y podría discutirse caso a caso), RN en estado crítico, con riesgo vital inminente y que sea considerado, por el neonatólogo a cargo, que es improbable que se beneficie de cuidados intensivos neonatales y falta de disponibilidad de equipo esencial, o de profesionales capacitados y en número sufi- 
ciente. En este caso se requiere traslado a centro que ofrezca la terapia. Los pacientes con malformación congénita severa o cromosomopatía, deben analizarse caso a caso, considerando proporcionalidad terapéutica.

Los puntos relevantes de la neuroprotección incluyen el que la hipotermia debe iniciarse tan pronto como sea posible ${ }^{20}$ (lo ideal sería antes de $3 \mathrm{~h}$ de vida), que frente a discrepancia entre los hallazgos en el aEEG y examen neurológico, se debe decidir sobre la base de los hallazgos del examen físico (revisar interpretación del aEEG) y dado que esta terapia ha demostrado beneficio para el paciente asfixiado de término o cercano a término, no es necesario consentimiento especial para esta terapia.

\section{Técnica de hipotermia ${ }^{4-6}$}

La temperatura debe ser controlada en forma continua con transductor esofágico, ubicado en su tercio medio comprobado por radiografía de tórax o en su defecto medición de temperatura rectal.

La hipotermia debe ser iniciada dentro de las $6 \mathrm{~h}$ del nacimiento, idealmente antes de las $3 \mathrm{~h}$ de vida y la duración debe ser de 72 h. Al término de las $72 \mathrm{~h}$ de hipotermia, la temperatura debe ir aumentando lentamente a no más de $0,5^{\circ} \mathrm{C}$ por $\mathrm{h}$, hasta $\operatorname{los} 36,5^{\circ} \mathrm{C}$ (normotermia). Una vez alcanzada la normotermia, la temperatura debe ser cuidadosamente monitoreada por lo menos durante $6 \mathrm{~h}$ para evitar hipertermia de rebote, ya que ésto podría ser perjudicial.

No hay evidencia para apoyar la seguridad $y$ el beneficio de un recalentamiento excesivamente lento, durante más de $12 \mathrm{~h}$, por lo que debe ser evitado.

\section{Métodos y temperatura objetivo}

La hipotermia moderada corporal total es la modalidad más estudiada. Deben ser utilizados dispositivos de hipotermia moderada, equipados con una manta base de temperatura servocontrolada. Están descritos otros dispositivos, tales como botellas de agua ${ }^{21,22}$, ventilador de aire $^{23}$, bolsas de gel refrigerado ${ }^{6}$.

El objetivo fundamental de la terapia es mantener la temperatura en forma estricta dentro de los límites específicos durante todo el período de hipotermia. Lo óptimo es realizar la terapia mediante el uso de una manta de agua con temperatura servocontrolada que circula permanentemente, sobre la que se recuesta al neonato.

El Protocolo ICE Trial ${ }^{6}$, realizado en 28 centros de Australia, Nueva Zelandia, Canadá, y USA, utilizó un sistema no servocontrolado con aplicación cerca del paciente de bolsas de gel refrigerado, no en contacto directo, demostrando lograr temperatura objetivo estable. Para esto se requiere alta dedicación de matrona o enfermera de intensivo neonatal calificada, durante las $72 \mathrm{~h}$ de terapia y las $12 \mathrm{~h}$ posteriores. Esta técnica de hipotermia no servocontrolada es replicable en forma protocolizada.

La temperatura objetivo corresponde a $33,5 \pm 0,5^{\circ} \mathrm{C}\left(33,0-34,0^{\circ} \mathrm{C}\right)$ medida a nivel esofágico y se debe alcanzar a través de enfriamiento de cuerpo entero.

\section{Control de temperatura}

La temperatura se monitorizará en forma continua usando sensor esofágico, ubicado en tercio medio del esófago. Se requiere control radiológico para confirmar ubicación. Mantener con óptima fijación para evitar desplazamientos.

Todas las decisiones clínicas se realizarán usando la temperatura esofágica. Además, se instalará un segundo sensor de temperatura en ubicación rectal, que actúa como medida de seguridad y que provea de control continuo en caso de mal funcionamiento del sensor esofágico. La temperatura esofágica se registrará cada $15 \mathrm{~min}$ en las primeras $2 \mathrm{~h}$, cada $30 \mathrm{~min}$ entre las 2 y $4 \mathrm{~h}$ y cada $1 \mathrm{~h}$ entre las 4 y $72 \mathrm{~h}$.

Control de frecuencia cardiaca (FC), presión arterial (PA) y frecuencia respiratoria (FR)

El límite de alarma inferior de FC en monitor será establecido en 90 latidos por min. Es tolerable bradicardia de hasta 70 latidos por minutos, siempre que el $\mathrm{RN}$ mantenga ritmo sinusal normal, PA estable y saturación adecuada.

El registro de FC, PA y FR, se realizará cada 15 min. entre las 0 y $2 \mathrm{~h}$, cada $30 \mathrm{~min}$. entre las 2 y $4 \mathrm{~h}$ y cada $1 \mathrm{~h}$ entre las 4 y $72 \mathrm{~h}$.

Algunos puntos importantes a considerar durante el período de hipotermia son que la 
temperatura de los RN sometidos a esta terapia se vuelve muy sensible a los cambios térmicos ambientales, debe evitarse la estimulación innecesaria y guardar silencio tanto como sea posible y la bradicardia es aceptable siempre y cuando la presión arterial y saturación se mantenga dentro del rango normal.

\section{Periodo de recalentamiento}

Durante el período de recalentamiento debe registrarse la temperatura esofágica y de piel cada $30 \mathrm{~min}$, hasta que la temperatura esofágica alcance $\operatorname{los} 36,5^{\circ} \mathrm{C}$. La FC, FR, y PA cada 1 hrs. hasta 6 horas después que la normotermia $\left(36,5^{\circ} \mathrm{C}\right)$ haya sido alcanzada (finalización de recalentamiento), manteniendo durante todo este tiempo monitoreo continuo de temperatura esofágica, con el fin de evitar rebote de temperatura.

\section{Eventos adversos y seguimiento durante la hipotermia.}

Debe realizarse un seguimiento cuidadoso de efectos adversos como hipotensión, arritmia, trombocitopenia, alteración de glicemia y signos de hipertensión pulmonar.

Los $\mathrm{RN}$ que requieren $\mathrm{FiO}_{2}>50 \%$ previo al inicio de la hipotermia, deben ser monitorizados cuidadosamente y se deben realizar los esfuerzos necesarios para optimizar la condición de ventilación y perfusión pulmonar, evaluar la presencia de una posible hipertensión pulmonar asociada y determinar si el enfriamiento aumenta aún más la necesidad de $\mathrm{O}_{2}$, en cuyo caso, el clínico debe considerar traslado a centro que disponga de óxido nítrico ${ }^{24}$.

La hipotermia puede aumentar el riesgo de úlceras por presión, por lo que es necesario establecer medidas de prevención con cambios frecuentes de puntos de apoyo y evitar el apoyo en pliegues, entre otras medidas de enfermería. Se sugiere realizar cambio de posición cada 6 $h$, movilizando en bloque manteniendo siempre la nariz alineada con el resto del cuerpo.

El equipo tratante debe considerar la probable suspensión de la hipotermia en caso de requerimiento de oxígeno $>90 \%$, hemorragia descontrolada, arritmia severa y/o sepsis grave. En todos estos casos se debe considerar un cuidadoso balance entre riesgos y beneficios.

\section{Evaluación con exámenes de laboratorio.}

Se monitorizará, a lo menos cada 12 h: glicemia, calcio iónico, electrolitos plasmáticos y gases arteriales. Se debe destacar que los gases están medidos en laboratorio a $37^{\circ} \mathrm{C}$, por lo cual la $\mathrm{pCO}_{2}$ aceptada en hipotermia de $33,5^{\circ} \mathrm{C}$ debe ser $45-50 \mathrm{~mm} \mathrm{Hg}$ (se multiplica por factor 0,83$)^{25}$.

Cada $24 \mathrm{~h}$ se deben agregar evaluaciones de pruebas hepáticas, estudio de coagulación, nitrógeno ureico, magnesio, fósforo, orina completa, recuento de plaquetas, enzimas cardíacas, ácido láctico y siempre descartar infección como patología asociada a asfixia.

Una vez alcanzada la temperatura objetivo de recalentamiento $\left(36,5^{\circ} \mathrm{C}\right.$.) se deben evaluar los siguientes exámenes: glicemia, gases, calcio iónico, nitrógeno ureico, creatininemia, electrolitos plasmáticos, pruebas de coagulación, hemograma con recuento de plaquetas y acido láctico.

\section{Accesos vasculares}

Es importante disponer de vías centrales, de preferencia catéter umbilical venoso y arterial. De no ser posible, se debe considerar una línea arterial periférica y una vía venosa periférica.

Se debe instalar los catéteres antes de iniciar hipotermia, debido a que la vasoconstricción por hipotermia producirá mayor dificultad técnica.

\section{Monitoreo EEG de amplitud integrada}

Además de la observación clínica cuidadosa y continua, es deseable el monitoreo electrofisiológico con aEEG, pues las convulsiones son comunes durante el período de hipotermia $^{26}$. El aEEG tiene menor sensibilidad para la detección de convulsiones que EEG convencional ${ }^{27}$. Se han realizado estudios sobre el valor pronóstico exacto de aEEG y EEG convencional durante la hipotermia ${ }^{28}$ apoyando el uso de aEEG.

\section{Medicación}

Se utiliza sedación, comenzando con infusión continua a dosis bajas con morfina o fentanilo, para evitar la estimulación simpática y el disconfort de la terapia ${ }^{29}$. Se deben evitar los escalofríos. 
No deben utilizarse relajantes musculares durante la hipotermia, dado que no ofrecen beneficios y pueden enmascarar crisis convulsivas clínicas.

Es razonable el uso de medicamentos antiepilépticos como el fenobarbital para manejo inicial de convulsiones neonatales. Frente a la menor sospecha de convulsión, iniciar terapia con fenobarbital. Otros medicamentos anticonvulsivantes se utilizarán según necesidad. La presentación clínica de las convulsiones puede ser variable ${ }^{30}$ y no siempre están acompañados de correlación electroencefalográfica ${ }^{31}$. El uso de fenobarbital profiláctico es controvertido ${ }^{32}$.

Es importante considerar que en hipotermia la vida media de los fármacos se alarga, por lo cual es ideal monitorizar los niveles de drogas, especialmente el fenobarbital.

\section{Eventos esperables durante la hipotermia ${ }^{29}$}

Durante la hipotermia puede presentarse disminución de FC, aumento de PA por vasoconstricción periférica, temblores, aumento en la diuresis debido a distribución de circulación a territorio esplácnico, disminución en la calcemia, fosfemia, magnesemia y kalemia, labilidad de la glicemia (por resistencia relativa a insulina, disminución de la tasa metabólica y temblores) y prolongación de las pruebas de coagulación en un $30 \%$.

Durante el recalentamiento es esperable encontrar recuperación de $\mathrm{FC}$, disminución en la PA (por disminución en la resistencia periférica), disminución en la diuresis (por aumento de tercer espacio y perfusión periférica) y convulsiones.

\section{Traslado}

En todo centro de atención que no cuente con UCIN, una vez determinada la presencia de asfixia neonatal, debe realizarse estabilización del paciente asegurando a lo menos vía venosa periférica o central para mantención de glicemia óptima y asegurar aporte de volumen durante reanimación y/o después de los primeros minutos de vida. Debe mantener permanentemente apagada la cuna calefaccionada.

Si es posible, apoyo con drogas vasoacti- vas, iniciando con dopamina a 5 gamma $/ \mathrm{kg} /$ min por vía central o si no se dispone de esta vía, utilizar por vía periférica dobutamina a igual dosis.

Las medidas anteriores no deben retrasar el contactarse con el centro de derivación e iniciar el proceso necesario para el traslado.

La temperatura rectal objetivo de traslado es de $34,5^{\circ} \mathrm{C}$. El traslado ${ }^{33,34,35}$ debe realizarse en incubadora apagada, con manguillas abiertas para mantener temperatura objetivo. Si con estas mediadas no se logra mantener temperatura objetivo, adicionar unidades refrigerantes (ice pack) en los bordes de la incubadora procurando que nunca tome contacto directo con la piel del paciente.

Se debe mantener un control estricto de temperatura, idealmente con monitor, y de no ser ésto posible controlar la temperatura rectal a lo menos cada $15 \mathrm{~min}$, para ello se debe disponer de termómetros especiales que marquen temperatura menor de $35^{\circ} \mathrm{C}$.

\section{Neuroimágenes}

La resonancia nuclear magnética (RNM) es preferible a la tomografía computarizada (TAC) o ecografía cerebral para la evaluación de las lesiones isquémicas.

La ecografía cerebral juega un importante rol en los primeros días de vida, especialmente durante períodos inestables y/o de hipotermia, entregando información, en la misma cuna del enfermo, sobre hemorragias, lesiones de núcleos basales y con doppler cerebral evidenciar signos de vasodilatación cerebral y/o perdida de la regulación del flujo cerebral.

Mientras el paciente no haya salido de su condición de terapia aguda, no se realiza estudio de neuroimágenes con TAC ni RNM, si no existe sospecha de urgencia neuroquirúrgica. Lo ideal es realizar RNM cerebral con espectroscopía; sólo ante imposibilidad de realizar RNM o por sospecha de urgencia neuroquirúrgica se puede realizar TAC cerebral. El estudio de neuroimágenes se debe realizar entre $\operatorname{los} 5^{\circ}$ y $14^{\circ}$ días de vida, estando el paciente en condición estable. La RNM por difusión también debe realizarse si se dispone de esta 
modalidad ${ }^{36}$. La espectroscopia por resonancia magnética (MRS) es el predictor más sensible de resultado en los neonatos con encefalopatía, por lo tanto se debe considerar adicionar a RNM, si ello es posible ${ }^{37}$.

\section{Estudio Anatomopatológico}

En caso de muerte, la autopsia después de obtenido el consentimiento de los padres es importante. Por ejemplo, la aparición de la lesión cerebral puede ser sugerida por el grado de reacciones gliales en comparación con la edad postnatal ${ }^{38,39}$.

\section{Seguimiento}

A pesar de que la hipotermia ha demostrado ser eficaz, sólo determina mejoría en un $20-25 \%$ de los niños en situación de riesgo. El número de pacientes necesario a tratar para prevenir la muerte o déficit neurológico grave a los 18 meses de edad es de 9, bajo el protocolo estándar actual. El grupo de niños con indicación de hipotermia sigue presentando muy alto riesgo de secuelas neurológicas, incluso después del tratamiento.

La atención multidisciplinaria con la participación de pediatras generales de atención secundaria, neonatólogos, neurólogos, terapeutas ocupacionales, kinesiólogos, fisiatras y psicólogos es fundamental para el seguimiento a largo plazo. Pruebas neurológicas y psicológicas estandarizadas como Bayley-III son preferibles desde el punto de vista de las comparaciones internacionales ${ }^{40}$.

\section{Criterios Institucionales}

Las instituciones dispuestas a ofrecer hipotermia para el tratamiento de EHI deben reunir las siguientes condiciones ${ }^{39}$ : corresponder a nivel III de complejidad, es decir disponer de camas de cuidados intensivos neonatales (UCIN); disponer de matrona o enfermera altamente capacitada, idealmente como mínimo una matrona o enfermera para 2 neonatos, du- rante las $72 \mathrm{~h}$ de hipotermia servocontrolada; en caso de instalar hipotermia con método no servocontrolado debe haber una profesional matrona o enfermera exclusiva, hasta $6 \mathrm{~h}$ una vez concluido recalentamiento; ser capaces de realizar estudio de neuroimagen, idealmente resonancia magnética, entre el $5^{\circ}$ y $14^{\circ}$ días después del evento; disponer de estudio con EEG, e idealmente tener capacidad de monitorizar con electroencefalograma de amplitud integrada (aEEG), así como neonatólogos capaces de realizar lectura de EEG de amplitud; ofrecer un enfoque multidisciplinario en el seguimiento a largo plazo de los pacientes, con un equipo formado por pediatras, neurólogo infantil, psicólogo y profesionales especialistas en rehabilitación; ser capaces de realizar estudios anatomopatológicos, como autopsia, estudios de específicos de patologías neurológicas y de placenta; realizar auditoría perinatal contemporánea de todos los casos de EHI generados in situ y en su red de responsabilidad y vigilar permanente incidencia de EHI, Apgar $5 \min \leq 6$ y muerte asociada a asfixia neonatal.

\section{Discusión}

La guía práctica de esta recomendación para el tratamiento de la EHI se basa en evidencia científica publicada a partir de los últimos grandes ensayos clínicos.

Es fundamental establecer las terapias de rescate ya descritas, nunca perdiendo el objetivo de generar todas las medidas de apoyo que favorezcan la oxigenación y perfusión cerebral y sistémica, asegurando óptima homeostasis celular ya que constituyen la base de las medidas de rescate del neonato asfixiado. Sobre estas terapias de rescate ya establecidas, instaurar la medida de neuroprotección más beneficiosa hasta hoy conocida, que es la hipotermia.

Con el fin de maximizar el beneficio para los pacientes asfixiados que están en alto riesgo, es muy importante para nosotros seguir protocolos estándares, basado en la mejor evidencia científica disponible ${ }^{41}$. Debemos estar disponibles, una vez estandarizada la terapia integral del RN con EHI, a protocolos con tratamientos farmacológicos de neuroprotección que se adicionen a la hipotermia ${ }^{42}$. 
Hay varios aspectos únicos en la medicina perinatal en Chile como la distancia entre centros terciarios de salud, número reducido de camas UCIN, dificultad de transporte neonatal, escasa cantidad de equipos para hipotermia $\mathrm{y}$ un reducido número de monitores de $\mathrm{aEEG}$, además de la inexperiencia en su uso, todo lo cual genera problemas adicionales. Es fundamental equipar en el más breve plazo, a las Unidades de Nivel Terciario del país con equipos de hipotermia servocontrolada, capacitar en su uso y en el óptimo manejo del neonato asfixiado, nunca olvidando que la hipotermia es sólo una parte del tratamiento.

Así mismo es imprescindible establecer auditorias de todos los casos de EHI ocurridos localmente y en su red de responsabilidad, así como también de Apgar a los $5 \min \leq 6$ y muerte perinatal asociada a asfixia, realizadas por equipo perinatal ${ }^{43}$, con el objetivo de establecer políticas locales de prevención de asfixia. Por otra parte, es necesario como país, establecer una base única de resultado intrahospitalario y de seguimiento de estos pacientes para analizar y mejorar. Sugerimos realizar análisis comparativo de resultados de asfixia perinatal entre los centros, para lo cual esta Sociedad Científica abre sus puertas.

\section{Referencias}

1.- Graham EM, Ruis KA, Hartman AL, Northington FJ, Fox HE: A systematic review of the role of intrapartum hypoxia-ischemia in the causation of neonatal encephalopathy. Am J Obstet Gynecol 2008; 199: 587-95.

2.- Latorre R, Carrillo J, Yamamoto M, et al: Gobierno del parto en el Hospital Padre Hurtado: Un modelo para contener la tasa de cesárea y prevenir la encefalopatía hipóxico-isquémica. Rev. Chil Obst Ginecol 2006; 71 (3): 196-200.

3.- Gluckman PD Wyatt JS Azzopardi D et al: Selective head cooling with mild systemic hypothermia after neonatal encephalopathy: multicentre randomised trial. Lancet 2005; 365 (9460): 663-70.

4.- Seetha Shankaran MD, Abbot R. Laptook, et al for the National Institute of Child Health and Human Development (NICHD) Neonatal Research Network: Whole-Body Hypothermia for Neonates with HypoxicIschemic Encephalopathy. N Engl J Med 2005; 353: 1574-84.
5.- Azzopardi DV, Strohm B, Edwards AD, Dyet L, Halliday $H L$, Juszczak E, et al for the TOBY Study Group. Moderate hypothermia to treat perinatal asphyxial encephalopathy. N Engl J Med 2009; 361: 1349-58.

6.- Jacobs SE, Morley CJ, Inder TE, et al for Infant Cooling Evaluation Collaboration. Whole-body hypothermia for term and near-term newborns with hypoxicischemic encephalopathy: a randomized controlled trial. Arch Pediatr Adolesc Med 2011; 165 (8): 692-700.

7.- Edwards A, Brocklehurst P, Gunn A, et al: Neurological outcomes at 18 months of age after moderate hypothermia for perinatal hypoxic ischaemic encephalopathy: synthesis and meta-analysis of trial data. BMJ 2010; 340: 363

8.- Perlman JM, Wyllie J, Kattwinkel J, et al: Part 11: neonatal resuscitation: 2010 International Consensus on Cardiopulmonary Resuscitation and Emergency Cardiovascular Care Science with Treatment Recommendations. Circulation 2010; 122: S516-38.

9.- Volpe JJ: Neurología del recién nacido. $5^{\circ}$ Edición, 2008. Unidad III.

10.- Cloherty J, Eichenwald, Stark A: Manual de Neonatología. $6^{\circ}$ Edición, 2009. 512-22.

11.- Mena P, Mulhlhausen G, Novoa J, Vivanco G: Guías Nacionales de Neonatología, 2005. Ministerio de Salud.

12.- Liu J, Li J, Gu M: The correlation between myocardial function and cerebral hemodynamics in term infants with hypoxic-ischemic encephalopathy. J Trop Pediatr 2007; 53 (1): 44-8.

13.- Armstrong K, Franklin O, Sweetman D, et al: Cardiovascular dysfunction in infants with neonatal encephalopathy. Arch Dis Child (2011). doi:2 of 4 10.1136/ adc. 2011.214205

14.- Caresta E, Papoff P, Benedetti $S$, et al: What's new in the treatment of neonatal shock. The Journal of Maternal-Fetal and Neonatal Medicine 2011; 24 (S(1)): 17-9.

15.- Battin M, Thoresen $M$ and on behalf of the Cool Cap Trial Group: Does Head Cooling With Mild Systemic Hypothermia Affect Requirement for Blood Pressure Support? Pediatrics 2009; 123: 1031-6.

16.- Walid A, Salhab WA, MD; Myra H, Salhab WA, Wyckoff $M H$, Laptook AR, Perlman JM: Initial hypoglycemia and neonatal brain injury in term infants with severe fetal acidemia. Pediatrics 2004; 114: 361-6.

17.- Basu P Som S Choudhuri M Das H: Contribution of the blood glucose level in perinatal asphyxia. Eur J Pediatr 2009; 168 (7): 833-88.

18.- Kendall GS, Kapetanakis A, Ratnavel $M$ Azzopardi D Robertson N]: Cooling on Retrieval Study Group: Pas- 
sive cooling for initiation of therapeutic hypothermia in neonatal encephalopathy. Arch Dis Child Fetal Neonata Ed 2010; 95 (6): F408-12.

19.- Sarkar S, Barks JD, Donn SM: Should amplitudeintegrated electroencephalography be used to identify infants suitable for hypothermic neuroprotection? J Perinatol 2008; 28: 117-22.

20.- Gunn AJ: Cerebral hypothermia for prevention of brain injury following perinatal asphyxia. Curr Opin Pediatr 2000; 12: 111-5.

21.- Iwata $S$, Iwata $O$, Olson L, et al: Therapeutic hypothermia can be induced and maintained using either commercial water bottles or a "phase changing material" mattress in a newborn piglet model. Arch Dis Child 2009; 94: 387-91.

22.- Robertson NJ, Nakakeeto M, Hagmann C, et al: Therapeutic hypothermia for birth asphyxia in low-resource settings: a pilot randomised controlled trial. Lancet 2008; 372: 801-3.

23.- Horn A, Thompson C, Woods D, et al: Induced hypothermia for infants with hypoxic-ischemic encephalopathy using a servo-controlled fan: an exploratory pilot study. Pediatrics 2009; 123: e1090-8.

24.- Thoresen and Whitelaw. Cardiovascular Changes During Mild Therapeutic Hypothermia and Rewarming in Infants With Hypoxic-Ischemic Encephalopathy. Pediatrics 2000; 106: 92-9.

25.- Zanelli, et al: State Of The Art, Physiologic and pharmacologic considerations for hypothermia therapy in neonates. Journal of Perinatology 2011; 3: 377-86.

26.- Yap V, Engel M, Takenouchi T, Perlman JM: Seizures are common in term infants undergoing head cooling. Pediatr Neurol 2009; 41: 327-31.

27.- Shellhaas RA, Soaita AI, et al: Sensitivity of amplitud integrated electroencephalography for neonatal seizure detection. Pediatrics 2007; 120: 770-7.

28.- Thoresen M, Hellstrom-Westas L, Liu X, de Vries LS: Effect of hypothermia on amplitude-integrated electroencephalogram in infants with asphyxia. Pediatrics 2010; 126: e131-9.

29.- Thoresen M: Supportive Care During Neuroprotective Hypothermia in the Term Newborn: Adverse Effects and Their Prevention. Clin Perinatol 2008; 35: 749-63.

30.- Volpe JJ: Neonatal seizures: current concepts and revised classification. Pediatrics 1989; 84: 422-8.

31.- Scher MS, Aso K, Beggarly ME, Hamid MY, Steppe $D A$, Painter $M J$ : Electrographic seizures in preterm and full-term neonates: clinical correlates, associated brain lesions, and risk for neurologic sequelae. Pediatrics 1993; 91: 128-34.

32.- Hall RT, Hall FK, Daily DK: High-dose phenobarbital therapy in term newborn infants with severe perinatal asphyxia: a randomized, prospective study with threeyear follow-up. J Pediatr 1998; 132: 345-8.

33.- Robertson N Kendall GS Thayyil S. Techniques for therapeutic hypothermia during transport and in hospital for perinatal asphyxial encephalopathy. Semin Feta Neonatal Med 2010; 15 (5): 276-86.

34.- Anderson ME, Longhofer TA, Phillips W, McRay DE: Passive cooling to initiate hypothermia for transported encephalopathic newborns. Journal of Perinatology 2007; 27: 592-3.

35.- O'Reilly KM Tooley Winterbottom S. Therapeutic hypothermia during neonatal transport. Acta Paediat. 2011; 100 (8): 1084-6.

36.- Ment LR, Bada HS, Barnes P, et al: Practice parameter: neuroimaging of the neonate: report of the Quality Standards Subcommittee of the American Academy of Neurology and the Practice Committee of the Child Neurology Society. Neurology 2002; 58: 1726-38.

37.- Thayyil S, Chandrasekaran M, Taylor A, et al: Cerebral magnetic resonance biomarkers in neonatal encephalopathy: a meta-analysis. Pediatrics 2010; 125: e382-95.

38.- Armstrong D, Halliday W, Hawkins C, Takashima S: "Perinatal Brain Damage". In: "Pediatric Neuropathology”. New York: Springer; 2007. p. 83-109.

39.- Takenouchi $T$, et al: Therapeutic hypothermia for neonatal encephalopathy: JSPNM \& MHLW Japan Working Group Practice Guidelines. Brain Dev (2011), doi:10.1016/j.braindev.2011.06.009

40.- de Vries LS, Jongmans MJ: Long-term outcome after neonatal hypoxic-ischaemic encephalopathy. Arch Dis Child Fetal Neonatal Ed 2010; 95: F220-4.

41.- Higgins, et al: Hypothermia and Other Treatment Options for Neonatal Encephalopathy: An Executive Summary of the Eunice Kennedy Shriver NICHD Workshop. Journal of Pediatrics 2011; 159 (5): 851-8.

42.- Levene M: Cool treatment for birth asphyxia, but what's next? Arch Dis Child Fetal Neonatal Ed 2010; 95: F154-7.

43.- Novoa J, Milad M, Vivanco G, Fabres J, Ramírez R: Recomendaciones de organización, características y funcionamiento en Servicios o Unidades de Neonatología. Rev Chil Pediatr 2009; 80 (2): 168-87. 